IdeAs

Idées d'Amériques

$6 \mid 2015$

Migrer dans les Amériques

\title{
Migrer dans les Amériques
}

\section{Françoise Lestage et Paul Schor}

\section{OpenEdition}

\section{Journals}

Édition électronique

URL : https://journals.openedition.org/ideas/1038

DOI : 10.4000/ideas. 1038

ISSN : 1950-5701

Traduction(s) :

Migrating in the Americas - URL : https://journals.openedition.org/ideas/3084 [en]

Migrar en las Américas - URL : https://journals.openedition.org/ideas/3091 [es]

Migrar nas Américas - URL : https://journals.openedition.org/ideas/4728 [pt]

\section{Éditeur}

Institut des Amériques

\section{Référence électronique}

Françoise Lestage et Paul Schor, « Migrer dans les Amériques », IdeAs [En ligne], 6 | 2015, mis en ligne le 09 décembre 2015, consulté le 19 octobre 2022. URL : http://journals.openedition.org/ideas/1038 DOI : https://doi.org/10.4000/ideas.1038

Ce document a été généré automatiquement le 19 octobre 2022.

\section{(c) $(1) \&$}

Creative Commons - Attribution - Pas d'Utilisation Commerciale - Pas de Modification 4.0 International - CC BY-NC-ND 4.0

https://creativecommons.org/licenses/by-nc-nd/4.0/ 


\title{
Migrer dans les Amériques
}

\author{
Françoise Lestage et Paul Schor
}

1 Consacré à la migration dans les Amériques, ce dossier est dédié à la mémoire d'Aristide Zolberg (1931-2013), dont les travaux de déconstruction du mythe des Etats-Unis, terre de migrants ${ }^{1}$, ont constitué un apport important à l'étude des migrations et de la construction nationale, et qui participa au colloque «La migration en héritage » à Paris en juin 2012 où plusieurs textes inclus dans ce dossier avaient été discutés².

2 Si la migration est un phénomène global, les Amériques entretiennent une relation particulière avec elle, puisque le mot même de "migration" est contemporain des premières mobilités transatlantiques qui suivent la « découverte » de l'Amérique à la fin du XVe siècle. Les Amériques - dont l'assignation identitaire de " Nouveau Monde » rend déjà compte d'une temporalité que traversent les expériences migratoires - ont connu depuis des vagues multiples de flux migratoires, transocéaniques et intraaméricains, faisant de ce continent le lieu de rencontre, d'installation, de confrontation mais également d'échange des populations issues des différentes parties du monde. A l'échelle du continent, force est de constater «la longue durée» du phénomène migratoire, pour ne pas dire son caractère constitutif. L'Amérique et son peuplement largement venu d'ailleurs ont en quelque sorte reçu « la migration en héritage ».

Pour aborder un thème aussi central, les auteurs rassemblés ici ont mobilisé les méthodes et les ressources de plusieurs disciplines, histoire, géographie, anthropologie, sociologie, jouant avec les échelles et les temporalités, afin d'approcher non pas une image exhaustive de la migration dans les Amériques, ce qui serait impossible, mais la manière dont le présent et le passé des migrations affectent les expériences vécues des populations migrantes et de leurs descendants et, plus largement, des sociétés d'origine et d'accueil. Les études migratoires, en particulier dans les Amériques, ont été profondément renouvelées depuis leurs origines et il est parfois malaisé de redonner une cohérence à ce phénomène qui est aujourd'hui appréhendé sous de multiples facettes. C'est pourtant l'ambition de ce dossier. L'hétérogénéité voulue des contributions permet de rendre compte de la diversité des mémoires, des expériences et des circulations, mais aussi de faire tenir ensemble des travaux qui partagent une approche centrée sur la personne, l'individu et l'expérience humaine, directe ou 
indirecte, de la migration. A travers les correspondances, les archives, les enquêtes, les chiffres et les terrains, se dessine la figure de migrants en mouvement qui, par leurs déplacements, leurs traces et leurs connexions donnent sa spécificité au continent américain.

Fondatrice des Amériques, la migration participe aussi d'un système global, et le passage d'une échelle à une autre permet d'inscrire les différents cas dans l'histoire et l'actualité de la mondialisation. Qu'ils s'agisse de travailleurs pauvres, de migrants qualifiés, de militants ou d'entrepreneurs, allant de trajectoires bien définies à des pratiques de circulations complexes entre régions et pays, les déplacements des migrants mettent en relations des pays et des zones différentes, mais de plus en plus interconnectées. De ce point de vue, pris ensemble, les articles de ce dossier - qui dans leur majorité s'intéressent à des cas nationaux - permettent aussi de voir que les parcours migratoires dessinent la géographie du continent, au point que tous sont affectés, y compris ceux qui ne migrent pas.

5 Malgré la diversité des sujets traités, les auteurs se retrouvent dans des thèmes généraux qui traversent les écrits. Le premier est celui de la mémoire vivante de la migration, de la continuité de l'expérience vécue, bien au-delà du moment de la migration stricto sensu, notamment à travers l'étude des correspondances, un matériau qui a connu récemment un renouveau historiographique. Les usages différenciés de la correspondance par les migrants et les voyageurs montrent comment se font et se défont, dans la durée, les réseaux transnationaux. Qu'il s'agisse de l'étude d'Aubert qui étudie comment, par l'écrit et la photographie, les missionnaires épiscopaliens ont produit une image littéraire et visuelle de leurs «terrains » du sud du continent, du Brésil au Chili, dans un double mouvement d'exoticisation et d'intégration au projet impérial états-unien, ou qu'il s'agisse des lettres de familles de migrants siciliens en Argentine étudiées par Da Orden sur plusieurs générations (1910-1980), la correspondance permet de reconstituer la temporalité des expériences migratoires. Dans le cas de Da Orden, on voit comment la correspondance remplit plusieurs fonctions et devient, pour l'historien, le support de l'étude des relations de pouvoir au sein des familles et des reconfigurations des relations familiales dans le contexte migratoire. A son tour, Venkovits analyse comment la correspondance et les récits de voyageurs hongrois ont pu être encouragés et orientés par le gouvernement de Porfirio Díaz pour les valoriser auprès de potentiels migrants européens vers le Mexique au détriment des Etats-Unis.

6 La mémoire de la migration, qu'elle s'inscrive dans un projet politique ou une stratégie familiale étendue à la parentèle s'exprime non seulement par les écrits mais aussi par une patrimonialisation qui passe par les objets, d'abord transmis au sein des familles puis rassemblés dans des musées, comme le montre Daniel pour les descendants d'immigrés finlandais, juifs ou allemands aux Etats-Unis. En donnant aux objets et archives de familles une place dans des musées ethniques, ils en font à la fois une institution pour le groupe et inscrivent symboliquement les communautés dans le pays d'accueil. Cette ethnicité symbolique, produite par les générations suivantes, situe elle aussi la migration dans une temporalité longue, multigénérationnelle, où histoire et mémoire sont enchevêtrées.

7 On le voit, quant il est question de migration, les allers-et-retours entres les espaces et les périodes sont multiples et complexes. Cela est manifeste lorsqu'on appréhende l'histoire longue des migrations, mais ce va-et-vient est aussi à l'œuvre dans les cas plus 
contemporains qui figurent dans ce dossier. En analysant les comportements des migrants endeuillés au Québec dans les années 2010, Rachedi et al. concluent à une large palette de comportements qui vont de la volonté de répliquer à l'identique les rituels funéraires des ancêtres à la modification, voire l'invention, de rites inédits permettant aux migrants et à leurs descendants de se projeter vers l'avenir dans la société où ils vivent.

8 Le deuxième thème est celui de l'expérience migratoire qui constitue, on l'a dit, un point de vue récent dans les travaux sur les migrations. Plusieurs auteurs envisagent cette question sous l'angle de la reconnaissance sociale, les migrants souhaitant obtenir des droits et un statut en accord avec leur participation économique ou politique dans la société d'accueil. Schaffhauser s'attache aux mobilisations des journaliers agricoles mexicains des années 40 à 60 , les braceros, qui dans les années 2000 se mettent à revendiquer une retraite pour laquelle ils ont cotisé mais qui ne leur a pas été payée ; Luconi compare la participation politique des migrants italiens en Argentine et aux Etats-Unis dans la première moitié du $20^{\text {ème }}$ siècle.

9 Les conclusions des deux auteurs divergent: Schaffhauser voit dans la récente expérience politique des retraités braceros un apport démocratique à la société mexicaine contemporaine : pour cet auteur, la demande des Braceros, qui croient fermement que leurs droits seront reconnus et se mobilisent dans ce sens sans faillir, « réinstitutionnalise » les institutions mexicaines en les contraignant à agir comme un service public, ce qui est loin d'être toujours le cas au Mexique. Pour Luconi, selon que les migrants italiens se sont installés aux Etats-Unis, déjà dominés par les protestants nord-européens, ou en Argentine, où la société nationale était encore à construire, ils ont été acceptés plus ou moins tardivement dans l'arène politique et ont pu, ou pas, y jouer un rôle. En Argentine, l'élection du premier président d'origine italienne en 1890 a marqué indubitablement cette reconnaissance, qui fut bien plus tardive aux EtatsUnis.

Projet éminemment politique, l'émigration volontaire de Noirs américains vers Haïti avant l'abolition de l'esclavage montre comment ceux qui partent (et parfois reviennent), tout comme ceux qui envisagent de partir mais restent, participent à des constructions politiques nationales complexes, celle de Haïti ainsi que celle des EtatsUnis. Bourhis-Mariotti illustre cet aspect peu connu de l'histoire de l'abolitionnisme où la migration, comme projet ou comme réalité, constitue des sujets politiques, à rebours des tendances à l'œuvre aux Etats-Unis dans les dernières années de l'esclavage. La migration volontaire, pour les descendants d'esclaves, à la différence des projets d'émigration d'affranchis voulus par les abolitionnistes blancs, apparait alors comme une affirmation de liberté et une critique en actes du racisme du régime politique étatsunien.

11 Cohen déplace la focale au niveau de l'intime, en montrant comment les travailleurs mexicains sans papiers d'un restaurant de Chicago où elle a fait une observation participante, entretiennent simultanément des relations amoureuses et familiales, dont le statut est différent, des deux côtés de la frontière. Ici la frontière structure des relations amoureuses marquées par l'inégalité entre les sexes, mais aussi, à Chicago, par une mixité ethnique et sociale (les maîtresses n'appartiennent pas au groupe et sont plus éduquées) que les hommes disent sans conséquence. Les relations asymétriques entre hommes migrants et femmes américaines complexifient les hiérarchies de statuts et de pouvoir entre migrants et non-migrants et l'on voit 
comment la situation de migrant peut réordonner les arrangements les plus personnels.

Un troisième thème du dossier permet de mesurer comment la circulation des personnes unit les espaces, que ce soit sur le continent américain (Morales, BabyCollin), entre les Amériques et l'Europe (Gérard etGrediaga et Miret et Cordoba) ou entre l'Asie et les Amériques (Essses, Medianu et Sutter), en partant d'objets très divers telle la mobilité des élèves ingénieurs mexicains ou le rapport à l'espace vécu des Colombiens de Barcelone.

Pour Gérard et Grediaga, qui étudient les parcours de formation des élèves ingénieurs mexicains pendant la seconde moitié du $20^{\text {ème }}$ siècle, et qui interrogent l'articulation entre les mobilités et la circulation des savoirs dans un monde globalisé et hiérarchisé, les mobilités ont des effets paradoxaux : ils constatent que la construction de « chaînes de savoirs ", autrement dit de coopérations privilégiées entre institutions mexicaines et étrangères, initialement liée à la mobilité des élèves, réduit cette mobilité de génération en génération. Ils remarquent aussi que les trajectoires de formation se sont modifiées, créant de nouveaux rapports institutionnels et de nouveaux circuits de mobilité, rompant ainsi avec certains héritages.

Les transformations de l'espace sont également un effet de la mobilité des personnes, remarquent Miret et Cordoba qui cherchent à comprendre comment les expériences urbaines dans le pays d'accueil ont une incidence sur les modifications de l'habiter dans le pays d'origine, en se fondant sur l'expérience biographique et le discours d'une vingtaine de Colombiens arrivés à Barcelone dans les années 2000, étudiants ou migrants qualifiés. Ces migrants, de retour en Colombie, adoptent de nouveaux modes de vie urbains, proches de ceux qu'ils ont connus à l'étranger. Inversement, Miret et Cordoba font l'hypothèse que la représentation négative de l'espace urbain local, en Colombie, et la représentation valorisée et mythifiée de l'espace urbain européen, peut avoir incité les mêmes Colombiens à migrer.

15 La diversification et la complexification des trajectoires migratoires influe à son tour sur les parcours et sur l'installation dans de nouvelles destinations comme l'étudie Baby-Collin en s'attachant à une migration peu connue, celle des Boliviens aux EtatsUnis, et en questionnant la part de cette destination dans le champ migratoire bolivien et dans le vécu des migrants.

Enfin, comme l'analysent Esses et al. dans le cas du Canada, la construction des perceptions des migrants est aussi façonnée par le traitement médiatique des "crises" migratoires qui intègrent tous les pays dans un système global de mobilités. Un système dans lequel s'inscrivent les pays d'Amérique centrale, objets de l'article de Morales qui décrit leur progressive états-unisation après les révolutions de la seconde moitié de $20^{\text {ème }}$ siècle, et leur intégration dans la globalisation économique, idéologique et politique par le biais de leurs migrants. En effet, ces derniers sont des acteurs éloignés de leurs sociétés d'origine, exclus, ou du moins tenus en-dehors de leurs systèmes sociaux, politiques et économiques. Mais ils sont aussi les pourvoyeurs d'une manne, les mandats envoyés à leurs proches, dont la distribution produit des recompositions sociales et territoriales dans la région. Ainsi Morales clôt ce dossier en reliant expérience migratoire individuelle, mobilités régionales, nationales et internationales, et globalisation. Pour lui, le «sujet migrant» constitue un maillon-clé dans les modifications politiques, économiques et sociales de l'Amérique centrale, tout comme il l'est pour les auteurs des articles de ce dossier. 
Partir de l'expérience des migrants pour mettre à jour les trajectoires, les réseaux, les ancrages et appartenances complexes, permet ainsi de dresser un autre portrait des Amériques, un continent où les mobilités structurent profondément les sociétés et les relations, individus, familles, groupes, régions et pays.

\section{NOTES}

1. Aristide R. Zolberg, A Nation by Design. Immigration Policy in the Fashioning of America, Russel Sage Foundation Books at Harvard University Press, 2008

2. 10ème colloque annuel de l'Institut des Amériques, organisé par une équipe de chercheurs de l'université Paris Diderot, https://sites.google.com/site/colloqueida2012/

\section{AUTEURS}

\section{FRANÇOISE LESTAGE}

Françoise Lestage est professeur des universités en anthropologie et sociologie à l'Université Paris Diderot, UMR URMIS (IRD-CNRS), où elle est co-responsable de l'axe de recherches « Les reconfigurations des migrations contemporaines ». Depuis septembre 2014, elle est détachée au Ministère des Affaires étrangères pour diriger le Centre d'études mexicaines et centraméricaines (CEMCA), Unité mixte CNRS des instituts français à l'étranger (UMIFRE 16) basée à Mexico.Elle est l'auteur de 3 livres en nom propre (1999, 2008, 2011), 2 livres comme coéditrice au Mexique (2011) et en France (2014), 4 directions de dossiers dans revues classées AERES (2009, 2012, 2013, 2014), 51 articles et chapitres de livres. Ces publications ont porté sur les politiques migratoires au Mexique depuis la fin des années 1990 ; sur les relations inter-ethniques en milieu migrant, sur les défunts dans la vie sociale et familiale des migrants; et sur le lien familial et le cycle de vie.Ses recherches actuelles se développent selon trois axes : la gestion publique des états d'affliction des migrants par l'Etat mexicain ; les pratiques et perceptions des « retours » des migrants au Mexique et en Amérique centrale ; les inégalités dans l'accès à la reproduction au Mexique et les circuits de maternité pour autrui.Elle a été membre du conseil scientifique de l'Institut des Amériques, et elle est, ou a été, membre des comités de rédaction des revues Problèmes d'Amérique Latine, Migraciones internacionales, Journal de la Société des Américanistes et Mexican Studies/Estudios Mexicanos.

\section{PAUL SCHOR}

Paul Schor est maître de conférences en histoire et civilisation américaines à l'Université Paris Diderot, UMR LARCA 8225, où il est responsable de l'axe de recherches « Histoire du politique dans le monde anglophone ». Il est l'auteur de Compter et classer. Histoire des catégories de la population dans le recensement américain, 1790-1940, Editions de l'EHESS, 2009, ouvrage récompensé 
par le Willi Paul Adams Award de l'Organization of American Historians, 2011 (traduction en langue anglaise sous presse à Oxford University Press, prévue courant 2016) et avec Nicolas Barreyre, De l'émancipation à la ségrégation: le Sud des États-Unis après la guerre de Sécession, 1865-1896, PUF, 2009.Il a publié de nombreux articles et chapitre d'ouvrages sur l'histoire des catégories raciales aux États-Unis, sur l'histoire de l'immigration aux États-Unis et sur l'histoire des ÉtatsUnis écrite en Europe, notamment Manfred Berg, Isabel Soto et Paul Schor, "The Weight of Words: writing about race in the United States and Europe", The American Historical Review, 119 (3), June 2014, p. 800-808.Ses recherches actuelles portent sur le rôle des experts et travailleurs sociaux dans l'imposition de nouvelles normes de vie quotidienne aux États-Unis au début du vingtième siècle.Il a été membre du conseil scientifique de l'Institut des Amériques et est membre des comités de rédaction des revues Sociétés contemporaines et Transatlantica. 